\title{
Implicit matrix representations of rational Bézier curves and surfaces
}

\author{
Laurent Busé \\ GALAAD, INRIA, 2004 route des Lucioles, 06902 Sophia Antipolis, France
}

\begin{abstract}
We introduce and study a new implicit representation of rational Bézier curves and surfaces in the 3-dimensional space. Given such a curve or surface, this representation consists of a matrix whose entries depend on the space variables and whose rank drops exactly on this curve or surface. Our approach can be seen as an extension of the moving lines implicitization method introduced by Sederberg, from non-singular matrices to the more general context of singular matrices. In the first part of this paper, we describe the construction of these new implicit matrix representations and their main geometric properties, in particular their ability to solve efficiently the inversion problem. The second part of this paper aims to show that these implicitization matrices adapt geometric problems, such as intersection problems, to the powerful tools of numerical linear algebra, in particular to one of the most important: the singular value decomposition. So, from the singular values of a given implicit matrix representation, we introduce a real evaluation function. We show that the variation of this function is qualitatively comparable to the Euclidean distance function. As an interesting consequence, we obtain a new determinantal formula for implicitizing a rational space curve or surface over the field of real numbers. Then, we show that implicit matrix representations can be used with numerical computations, in particular there is no need for symbolic computations to use them. We give some rigorous results explaining the numerical stability that we have observed in our experiments. We end the paper with a short illustration on ray tracing of parameterized surfaces.
\end{abstract}

Key words: Implicitization, moving planes, Bézier patches, inversion problem, intersection problems.

\section{Introduction}

In geometric modeling, parameterized algebraic curves and surfaces are used intensively. To manipulate them, it is useful to have an implicit representation, in addition to their given parametric representation. Indeed, a parametric representation is for instance well adapted for visualization purposes whereas an implicit representation allows significant improvements in the computation of intersections. Nevertheless, implicit representations are known to be very hard to compute in general. The goal of this paper is to overcome this difficulty by introducing a simple method for computing an implicit representation of a parameterized curve or surface in the form of a matrix. We will call them implicit matrix representations.

Matrix-based implicit representations of plane curves and surfaces already appeared several times in the literature (see e.g. $[12,13,23,25])$. However, all these approaches aimed at building a non-singular matrix whose determinant is an implicit polynomial equation. The case of plane curves is well understood: it is always possible to build such

Email address: Laurent.Buse@inria.fr (Laurent Busé). a non-singular matrix, in particular by means of the moving lines method introduced by Sederberg [25]. The case of surfaces is much more involved because of their rich geometry and the occurrence of base points (the points where the parameterization is not well defined). Thus, in order to find a non-singular matrix whose determinant is an implicit polynomial equation, one has to consider some very particular classes of parameterizations (see e.g. $[13,8,20]$ ).

In this paper, we show that matrix-based implicit representations can be built for (almost all) parameterized algebraic curves, including space curves, and surfaces if the requirement of getting a non-singular matrix is deleted. Indeed, the matrices we will introduce are in general singular matrices, but they still represent the curve or surface: the vanishing of a determinant will be replaced by a drop-ofrank property. Our approach is hence to keep these matrices as implicit representations on their own and to develop their study and use. Added and combined to the parametric representations, we believe that these implicit matrix representations can be a powerful tool.

The theoretical foundations of implicit matrix representations have been developed in a couple of papers $[9,6]$, using tools from algebraic geometry and commutative alge- 
bra. We will review their main properties in Section 3 . The first contribution of this paper is to adapt the construction of these matrices to the context of Bézier patches (usually obtained from the decomposition of a NURBS). This is the content of Section 2. The rest of the paper, and its main contribution, is a study of implicit matrix representations through a famous tool of numerical linear algebra: the singular value decomposition (SVD). It turns out that combined with this SVD, implicit matrix representations have very nice properties and show a very good numerical behavior. In Section 4, a real evaluation function is built from an implicit matrix representation and it is shown that this function is comparable to the classical Euclidean distance. As a byproduct, a new determinantal formula for implicitizing a rational Bézier curve or surface over the field of real numbers is obtained. Section 5 is devoted to the study of the numerical behavior of implicit matrix representations. In particular, we show that there is no need for symbolic computations to use them and we prove results that give insights for their numerical stability and robustness that we have observed in our experiments. Finally, the paper ends with a short discussion on the application of implicit matrix representations to intersection problems.

\section{Constructing a family of matrices attached to a parameterization}

In this section, we construct in a general framework a family of matrices that is attached to a given parameterization $\phi$. Consider a parameterization $\phi$ of a curve or surface

$$
s \in \mathbb{R}^{1} \text { or } \mathbb{R}^{2} \stackrel{\phi}{\rightarrow}\left(\frac{f_{1}(s)}{f_{0}(s)}, \frac{f_{2}(s)}{f_{0}(s)}, \frac{f_{3}(s)}{f_{0}(s)}\right) \in \mathbb{R}^{3}
$$

where $f_{0}, f_{1}, f_{2}$ and $f_{3}$ are polynomials in the parameter $s$ of degree $\leqslant d$. Notice that $s$ stands for a single parameter $t \in$ $\mathbb{R}$ if $\phi$ parameterizes a curve, or for a couple of parameters $(u, v) \in \mathbb{R}^{2}$ if $\phi$ parameterizes a surface. Similarly, the word degree means a single degree (for curves and triangular surfaces) or a bi-degree (for tensor-product surfaces).

Let $\mathcal{B}_{d}=\left\{\phi_{1}(s), \ldots, \phi_{n_{d}}(s)\right\}$ be a set of blending functions that form a basis of polynomials of degree at most $d$ in the parameter $s$. Then, the parameterization $\phi$ is of the form

$$
\phi(s)=\frac{\sum_{i=1}^{n_{d}} w_{i} \mathbf{b}_{i} \phi_{i}(s)}{\sum_{i=1}^{n_{d}} w_{i} \phi_{i}(s)}
$$

where the points $\mathbf{b}_{i}=\left(x_{i}, y_{i}, z_{i}\right) \in \mathbb{R}^{3}, i=1, \ldots, n_{d}$, are the control points and $w_{1}, \ldots, w_{n_{d}}$ their associated weights. It follows that

$$
\begin{array}{r}
f_{0}(s)=\sum_{i=1}^{n_{d}} w_{i} \phi_{i}(s), f_{1}(s)=\sum_{i=1}^{n_{d}} w_{i} x_{i} \phi_{i}(s), \\
f_{2}(s)=\sum_{i=1}^{n_{d}} w_{i} y_{i} \phi_{i}(s), f_{3}(s)=\sum_{i=1}^{n_{d}} w_{i} z_{i} \phi_{i}(s) .
\end{array}
$$

Now, we fix a non-negative degree $\nu$ and build a matrix $\mathbb{M}_{\nu}(\phi)$ as follows. Consider the set of 4 -tuples of polynomials $\left(g_{0}(s), g_{1}(s), g_{2}(s), g_{3}(s)\right)$ such that

$$
\operatorname{deg}\left(g_{i}(s)\right) \leqslant \nu \text { and } \sum_{i=0}^{3} g_{i}(s) f_{i}(s) \equiv 0 .
$$

It is clearly a vector space and computing one of its bases, say $L_{1}, \ldots, L_{r_{\nu}}$ amounts to solving a single linear system. Each $L_{j}$ being a 4 -tuple of polynomials $\left(g_{0}, g_{1}, g_{2}, g_{3}\right)$, it can be identified with the polynomial

$$
L_{j}(s ; X, Y, Z):=g_{0}(s)+X g_{1}(s)+Y g_{2}(s)+Z g_{3}(s) \text {. }
$$

Moreover, we have the freedom to choose an arbitrary basis of polynomials of degree $\leqslant \nu$ to express the $g_{i}$ 's. Let $\mathcal{B}_{\nu}^{\prime}=$ $\left\{\psi_{1}(s), \ldots, \psi_{m_{\nu}}(s)\right\}$ be such a basis, then we have

$$
\begin{array}{r}
L_{j}(s ; X, Y, Z)=\left(\sum_{i=1}^{m_{\nu}} \lambda_{0, i}^{(j)} \psi_{i}(s)\right)+\left(\sum_{i=1}^{m_{\nu}} \lambda_{1, i}^{(j)} \psi_{i}(s)\right) X+ \\
\left(\sum_{i=1}^{m_{\nu}} \lambda_{2, i}^{(j)} \psi_{i}(s)\right) Y+\left(\sum_{i=1}^{m_{\nu}} \lambda_{3, i}^{(j)} \psi_{i}(s)\right) Z
\end{array}
$$

where the $\lambda_{k, i}^{(j)}$ are real numbers. By rearranging (3), we get

$$
\begin{array}{r}
L_{j}=\sum_{i=1}^{m_{\nu}}\left(\lambda_{0, i}^{(j)}+\lambda_{1, i}^{(j)} X+\lambda_{2, i}^{(j)} Y+\lambda_{3, i}^{(j)} Z\right) \psi_{i}(s) \\
=\sum_{i=1}^{m_{\nu}} \Lambda_{i, j}(X, Y, Z) \psi_{i}(s)
\end{array}
$$

where $\Lambda_{i, j}(X, Y, Z)$ is a linear polynomial in $\mathbb{R}[X, Y, Z]$. The matrix $\mathbb{M}_{\nu}(\phi)$ is then defined as the $m_{\nu} \times r_{\nu}$-matrix whose entry $(i, j)$ is the linear polynomial $\Lambda_{i, j}(X, Y, Z)$ :

$$
\mathbb{M}_{\nu}(\phi):=\left(\begin{array}{cccc}
\Lambda_{1,1} & \Lambda_{1,2} & \cdots & \Lambda_{1, r_{\nu}} \\
\Lambda_{2,1} & \Lambda_{2,2} & \cdots & \Lambda_{2, r_{\nu}} \\
\vdots & \vdots & & \vdots \\
\Lambda_{m_{\nu}, 1} & \Lambda_{m_{\nu}, 2} & \cdots & \Lambda_{m_{\nu}, r_{\nu}}
\end{array}\right)
$$

A geometric interpretation of this construction is the following. For any specific value of the parameter $s_{0}$, the equation $L_{j}\left(s_{0} ; X, Y, Z\right)=0$ defines a plane in $\mathbb{R}^{3}$. When the parameter $s$ varies, this plane varies as well and the equation $L_{j}(s ; X, Y, Z)=0$ is hence called (after Sederberg and his co-authors; see e.g. [25,13]) a moving plane. Moreover, by definition of $L_{j}$, we have

$$
L_{j}\left(s ; \frac{f_{1}(s)}{f_{0}(s)}, \frac{f_{2}(s)}{f_{0}(s)}, \frac{f_{3}(s)}{f_{0}(s)}\right)=0
$$

which means that the plane corresponding to the given parameter $s_{0}$ always goes through the point $\phi\left(s_{0}\right)$ (if it is well-defined, i.e. $f_{0}\left(s_{0}\right) \neq 0$ ) that belongs to the curve or the surface parameterized by $\phi$. Therefore, $L_{1}, \ldots, L_{r_{\nu}}$ is a collection of $r_{\nu}$ moving planes and for any specific value $s_{0}$ of the parameter $s$, the $r_{\nu}$ corresponding planes all intersect at the point $\phi\left(s_{0}\right)$. As we will see in the sequel, under suitable hypothesis this point is actually their unique intersection point.

Before giving the main properties of the matrices $\mathbb{M}_{\nu}(\phi)$ in the next section, we give more details on their construction in three particular cases of interest in CAGD. 


\subsection{Rational Bézier curves}

Assume that $\phi$ parameterizes a rational Bézier curve. In this situation, the blending functions $\mathcal{B}_{d}$ are given by the Bernstein polynomials

$$
B_{i}^{d}(t)=\left(\begin{array}{l}
d \\
i
\end{array}\right) t^{i}(1-t)^{d-i}, \quad i=0, \ldots, d .
$$

Therefore, we have

$$
\phi: t \in \mathbb{R} \mapsto \frac{\sum_{i=0}^{d} w_{i} \mathbf{b}_{i} B_{i}^{d}(t)}{\sum_{i=0}^{d} w_{i} B_{i}^{d}(t)}=\left(\frac{f_{1}(t)}{f_{0}(t)}, \frac{f_{2}(t)}{f_{0}(t)}, \frac{f_{3}(t)}{f_{0}(t)}\right)
$$

where $b_{i}=\left(x_{i}, y_{i}, z_{i}\right), i=0, \ldots, d$ are the control points, and $w_{0}, \ldots, w_{d}$ are their weights.

Now, we fix a degree $\nu \geqslant 0$ and we seek 4-tuples of polynomials $\left(g_{0}, g_{1}, g_{2}, g_{3}\right)$ satisfying (2) in the Bernstein basis, that is to say, we choose $\mathcal{B}_{\nu}^{\prime}=\left\{B_{0}^{\nu}(t), \ldots, B_{\nu}^{\nu}(t)\right\}$ so that for all $j=0, \ldots, 3$

$$
g_{j}(t)=\sum_{i=0}^{\nu} \alpha_{j, i} B_{i}^{\nu}(t), \alpha_{j, i} \in \mathbb{R} .
$$

For that purpose, we form a matrix $\mathbb{S}_{\nu}$ as follows: the first column is filled with the coefficients of $B_{0}^{\nu}(t) f_{0}(t)$ in the Bernstein basis $\mathcal{B}_{\nu+d}=\left\{B_{0}^{\nu+d}(t), \ldots, B_{\nu+d}^{\nu+d}(t)\right\}$, the second column with the coefficients of $B_{1}^{\nu}(t) f_{0}(t)$ and so on until the column $\nu+1$ which is filled with the coefficients of $B_{\nu}^{\nu}(t) f_{0}(t)$. Then, we continue this way and add three other similar blocks of columns with the polynomials $f_{1}(t), f_{2}(t)$ and $f_{3}(t)$. The matrix $\mathbb{S}_{\nu}$ is hence a $(d+\nu+1) \times 4(\nu+1)$ matrix that satisfies the equality of matrices

$$
\begin{aligned}
& {\left[B_{0}^{\nu+d}(t) B_{0}^{\nu+d}(t) \cdots B_{\nu+d}^{\nu+d}(t)\right] \mathbb{S}_{\nu}=} \\
& \quad\left[B_{0}^{\nu}(t) f_{0}(t) \cdots B_{\nu}^{\nu}(t) f_{0}(t) B_{0}^{\nu}(t) f_{1}(t) \cdots B_{\nu}^{\nu}(t) f_{3}(t)\right] .
\end{aligned}
$$

Notice that this matrix is easily filled with simple computations on control points. Indeed, for any polynomial $f(t)=$ $\sum_{i=0}^{d} c_{i} B_{i}^{d}(t)$ and $j=0, \ldots, \nu$ we have

$$
B_{j}^{\nu}(t) f(t)=\sum_{i=0}^{d} \frac{\left(\begin{array}{c}
\nu \\
j
\end{array}\right)\left(\begin{array}{c}
d \\
i
\end{array}\right)}{\left(\begin{array}{c}
d+\nu \\
i+j
\end{array}\right)} c_{i} B_{i+j}^{d+\nu}(t)
$$

By definition of $\mathbb{S}_{\nu}$, any vector in its null space is of the form $\left[\alpha_{0,0}, \ldots, \alpha_{0, \nu}, \alpha_{1,0}, \ldots, \alpha_{1, \nu}, \alpha_{2,0}, \ldots, \alpha_{2, \nu}, \alpha_{3,0}, \ldots, \alpha_{3, \nu}\right]^{T}$ and yields a 4 -tuple of polynomials (7) satisfying (2). Therefore, the null space of $\mathbb{S}_{\nu}$ corresponds to a $4(\nu+1) \times r_{\nu^{-}}$ matrix of the form

$$
\operatorname{Null}\left(\mathbb{S}_{\nu}\right)=\left[\begin{array}{c}
M_{\nu, 0} \\
M_{\nu, 1} \\
M_{\nu, 2} \\
M_{\nu, 3}
\end{array}\right]
$$

where the block matrices $M_{\nu, 0}, M_{\nu, 1}, M_{\nu, 2}$ and $M_{\nu, 3}$ are of size $(\nu+1) \times r_{\nu}$. By definition of $\mathbb{M}_{\nu}(\phi)$, we have

$$
\mathbb{M}_{\nu}(\phi)=M_{\nu, 0}+X M_{\nu, 1}+Y M_{\nu, 2}+Z M_{\nu, 3} .
$$

Example 1 As a simple illustration, consider the cubic curve with $d=3, w_{i}=1$ for all $i=0, \ldots, 3$ and $\mathbf{b}_{0}=$ $(0,0,0), \mathbf{b}_{1}=(1 / 3,0,0), \mathbf{b}_{2}=(2 / 3,1 / 3,0), \mathbf{b}_{3}=(1,1,1)$. Then, choosing $\nu=1$ the matrix $\mathbb{S}_{1}$ is of size $5 \times 8$ and the computation of its null space yields the matrix

$$
\mathbb{M}_{1}(\phi)=\left[\begin{array}{ccc}
X+Y+Z & X+Y & X \\
-1+Z & -1+Y & -1+X
\end{array}\right] .
$$

Taking $\nu=2, \mathbb{S}_{2}$ is of size $6 \times 12$ and $\mathbb{M}_{2}(\phi)$ is equal to

$$
\left[\begin{array}{cccccc}
2 X+2 Y & -2 X-Y & 2 X+2 Y+2 Z & -3 X-2 Y-Z & -X & 2 X \\
-1+Y & 1 & -1+Z & 3 / 2 & 1 / 2 & X-1 \\
0 & -1+Y & 0 & -1+Z & X-1 & 0
\end{array}\right] .
$$

\subsection{Triangular rational Bézier surfaces}

A triangular rational Bézier surface of degree $d$ corresponds to a parameterization of the form

$$
\begin{aligned}
\phi:(u, v) \in \mathbb{R}^{2} \mapsto \frac{\sum_{i+j=0}^{d} w_{i, j} \mathbf{b}_{i, j} B_{i, j}^{d}(u, v)}{\sum_{i+j=0}^{d} w_{i, j} B_{i, j}^{d}(u, v)} \\
=\left(\frac{f_{1}(u, v)}{f_{0}(u, v)}, \frac{f_{2}(u, v)}{f_{0}(u, v)}, \frac{f_{3}(u, v)}{f_{0}(u, v)}\right)
\end{aligned}
$$

where for all pairs of integers $i, j$ such that $0 \leqslant i+j \leqslant d$, $b_{i, j}=\left(x_{i, j}, y_{i, j}, z_{i, j}\right)$ is a control point, $w_{i, j}$ its weight and

$$
\begin{aligned}
B_{i, j}^{d}(u, v)=\left(\begin{array}{c}
d \\
i, j
\end{array}\right) u^{i} v^{j}(1-u-v)^{d-i-j} \\
\quad=\frac{d !}{i ! j !(d-i-j) !} u^{i} v^{j}(1-u-v)^{d-i-j}
\end{aligned}
$$

the corresponding Bernstein polynomial. We proceed similarly to the previous case of curves. We fix an integer $\nu \geqslant 0$ and seek 4-tuples of polynomials $\left(g_{0}, g_{1}, g_{2}, g_{3}\right)$ in the Bernstein basis

$$
\begin{gathered}
\mathcal{B}_{\nu}^{\prime}=\left\{B_{0,0}^{\nu}(u, v), B_{0,1}^{\nu}(u, v), B_{0,2}^{\nu}(u, v) \ldots, B_{\nu, 0}^{\nu}(u, v)\right\}: \\
g_{j}(u, v)=\sum_{\substack{i+j=0 \\
i, j \geqslant 0}}^{\nu} \alpha_{j, i} B_{i, j}^{\nu}(u, v), \alpha_{j, i} \in \mathbb{R} .
\end{gathered}
$$

To compute them, we form the matrix $\mathbb{S}_{\nu}$ whose columns are filled with the coefficients of

$$
B_{i, j}^{\nu}(u, v) f_{k}(t), 0 \leqslant i+j \leqslant \nu, 0 \leqslant i, j, k=0,1,2,3
$$

in the Bernstein basis

$$
\mathcal{B}_{\nu+d}=\left\{B_{0,0}^{\nu+d}(u, v), B_{0,1}^{\nu+d}(u, v) \ldots, B_{\nu+d, 0}^{\nu+d}(u, v)\right\} .
$$

The matrix $\mathbb{S}_{\nu}$ has hence $\left(\begin{array}{c}d+\nu+2 \\ 2\end{array}\right)$ rows and $4 m_{\nu}=4\left(\begin{array}{c}\nu+2 \\ 2\end{array}\right)$ columns and it satisfies the equality of matrices

$$
\begin{gathered}
{\left[B_{0,0}^{\nu+d}(u, v) B_{0,1}^{\nu+d}(u, v) \cdots B_{\nu+d}^{\nu+d, 0}(u, v)\right] \mathbb{S}_{\nu}=} \\
{\left[B_{0,0}^{\nu}(u, v) f_{0} \cdots B_{\nu, 0}^{\nu}(u, v) f_{0} B_{0,0}^{\nu}(u, v) f_{1} \cdots B_{\nu, 0}^{\nu}(u, v) f_{3}\right] .}
\end{gathered}
$$

Notice that for any polynomial

$$
f(u, v)=\sum_{i+j=0}^{d} c_{i, j} B_{i, j}^{d}(u, v)
$$


and any pair of integers $0 \leqslant k+l \leqslant \nu, 0 \leqslant k, l$ we have

$$
B_{k, l}^{\nu}(u, v) f(u, v)=\sum_{\substack{i+j=0 \\
i, j \geqslant 0}}^{d} \frac{\left(\begin{array}{c}
\nu \\
k, l
\end{array}\right)\left(\begin{array}{c}
d \\
i, j
\end{array}\right)}{\left(\begin{array}{c}
d+\nu \\
i+k, j+l
\end{array}\right)} c_{i, j} B_{i+k, j+l}^{d+\nu}(u, v) .
$$

So, $\mathbb{S}_{\nu}$ can be filled by simple computations on the control points of the parameterization $\phi$. Now, by definition of $\mathbb{S}_{\nu}$ the computation of its null space returns a $4\left(\begin{array}{c}\nu+2 \\ 2\end{array}\right) \times r_{\nu^{-}}$ matrix of the form (8) where each matrix $M_{\nu, i}$ is of size $\left(\begin{array}{c}\nu+2 \\ 2\end{array}\right) \times r_{\nu}$. Finally, we get

$$
\mathbb{M}_{\nu}(\phi)=M_{\nu, 0}+X M_{\nu, 1}+Y M_{\nu, 2}+Z M_{\nu, 3} .
$$

Example 2 As a simple illustration, we consider a parameterization of the unit sphere with $d=2, w_{0,0}=1$, $w_{0,1}=1, w_{0,2}=2, w_{1,0}=1, w_{1,1}=1, w_{2,0}=2, \mathbf{b}_{0,0}=$ $(1,0,0), \mathbf{b}_{0,1}=(1,0,1), \mathbf{b}_{0,2}=(0,0,1), \mathbf{b}_{1,0}=(1,1,0)$, $\mathbf{b}_{1,1}=(1,1,1), \mathbf{b}_{2,0}=(0,1,0)$. Choosing $\nu=1$ we form the $10 \times 12$-matrix $\mathbb{S}_{\nu}$ and find the matrix

$\mathbb{M}_{1}(\phi)=\left[\begin{array}{cccc}Y & 1-X+Y+Z & -1+X-Y & 0 \\ Y & -2 X+2 Y & -1+X-Y+Z & -Y \\ -1-X+Y & -2 X & 2 X & Z\end{array}\right]$.

\subsection{Tensor-product rational Bézier surfaces}

A tensor-product rational Bézier surface of bi-degree $\left(d_{1}, d_{2}\right)$ corresponds to a parameterization of the form

$$
\phi:(u, v) \in \mathbb{R}^{2} \mapsto \frac{\sum_{i=0}^{d_{1}} \sum_{j=0}^{d_{2}} w_{i, j} \mathbf{b}_{i, j} B_{i}^{d_{1}}(u) B_{j}^{d_{2}}(v)}{\sum_{i=0}^{d_{1}} \sum_{j=0}^{d_{2}} w_{i, j} B_{i}^{d_{1}}(u) B_{j}^{d_{2}}(v)}
$$

where the $b_{i, j}$ 's are the control points and the $w_{i, j}$ 's their weights. Here again, we proceed as in the previous cases. However, in this case the degree is actually a bi-degree, that is to say a pair of integers that corresponds to the degree in the variable $u$ and the degree in the variable $v$. In the sequel, all the inequalities between these bi-degrees are understood component-wise.

So, let us fix a bi-degree $\nu=\left(\nu_{1}, \nu_{2}\right)$ and seek 4-tuples of polynomials $\left(g_{0}, g_{1}, g_{2}, g_{3}\right)$ in the tensor product Bernstein basis

$$
\begin{gathered}
\mathcal{B}_{\nu}^{\prime}=\left\{B_{0}^{\nu_{1}}(u) B_{0}^{\nu_{2}}(v), B_{0}^{\nu_{1}}(u) B_{1}^{\nu_{2}}(v), \ldots, B_{\nu_{1}}^{\nu_{1}}(u) B_{\nu_{2}}^{\nu_{2}}(v)\right\}: \\
g_{j}(u, v)=\sum_{i=0}^{\nu_{1}} \sum_{j=0}^{\nu_{2}} \alpha_{j, i} B_{i}^{\nu_{1}}(u) B_{j}^{\nu_{2}}(v), \alpha_{j, i} \in \mathbb{R} .
\end{gathered}
$$

Here again, we form the matrix $\mathbb{S}_{\nu}$ whose columns are filled with the coefficients of $B_{i}^{\nu_{1}}(u) B_{j}^{\nu_{2}}(v) f_{k}(u, v)$, in the Bernstein basis

$$
\mathcal{B}_{\nu+d}=\left\{B_{0}^{\nu_{1}+d_{1}}(u) B_{0}^{\nu_{2}+d_{2}}(v), \ldots, B_{\nu_{1}+d_{1}}^{\nu_{1}+d_{1}}(u) B_{\nu_{2}+d_{2}}^{\nu_{2}+d_{2}}(v)\right\} .
$$

Hence the matrix $\mathbb{S}_{\nu}$ has $\left(\nu_{1}+d_{1}+1\right)\left(\nu_{2}+d_{2}+1\right)$ rows and $4 m_{\nu}=4\left(\nu_{1}+1\right)\left(\nu_{2}+1\right)$ columns and it satisfies the equality of matrices

$$
\begin{gathered}
{\left[B_{0}^{\nu_{1}+d_{1}}(u) B_{0}^{\nu_{2}+d_{2}}(v) \cdots B_{\nu_{1}+d_{1}}^{\nu_{1}+d_{1}}(u) B_{\nu_{2}+d_{2}}^{\nu_{2}+d_{2}}(v)\right] \mathbb{S}_{\nu}=} \\
{\left[B_{0}^{\nu_{1}}(u) B_{0}^{\nu_{2}}(v) f_{0} \cdots B_{\nu_{1}}^{\nu_{1}}(u) B_{0}^{\nu_{2}}(v) f_{0} \cdots B_{\nu_{1}}^{\nu_{1}}(u) B_{\nu_{2}}^{\nu_{2}}(v) f_{3}\right]}
\end{gathered}
$$

Observe that for any polynomial

$$
f(u, v)=\sum_{i=0}^{d_{1}} \sum_{j=0}^{d_{2}} c_{i, j} B_{i}^{d_{1}}(u) B_{j}^{d_{2}}(v)
$$

and any pair of integers $k, l$ such that $0 \leqslant k \leqslant \nu_{1}$ and $0 \leqslant$ $l \leqslant \nu_{2}$, we have

$$
\begin{aligned}
& B_{k}^{\nu_{1}}(u) B_{l}^{\nu_{2}}(v) f(u, v)= \\
& \quad \sum_{i=0}^{d_{1}} \sum_{j=0}^{d_{2}} \frac{\left(\begin{array}{c}
\nu_{1} \\
k
\end{array}\right)\left(\begin{array}{c}
\nu_{2} \\
l
\end{array}\right)\left(\begin{array}{c}
d_{1} \\
i
\end{array}\right)\left(\begin{array}{c}
d_{2} \\
j
\end{array}\right)}{\left(\begin{array}{c}
\nu_{1}+\nu_{2} \\
i+k
\end{array}\right)\left(\begin{array}{c}
d_{1}+d_{2} \\
j+l
\end{array}\right)} c_{i, j} B_{i+k}^{\nu_{1}+d_{1}}(u) B_{j+l}^{\nu_{2}+d_{2}}(v) .
\end{aligned}
$$

The computation of the null space of $\mathbb{S}_{\nu}$ returns a $4\left(\nu_{1}+\right.$ 1) $\left(\nu_{2}+1\right) \times r_{\nu}$-matrix of the form (8) where each matrix $M_{\nu, i}$ is of size $\left(\nu_{1}+1\right)\left(\nu_{2}+1\right) \times r_{\nu}$. As in the previous cases, we get

$$
\mathbb{M}_{\nu}(\phi)=M_{\nu, 0}+X M_{\nu, 1}+Y M_{\nu, 2}+Z M_{\nu, 3} .
$$

Example 3 As a simple illustration, we consider a parameterization of a tensor product surface of bi-degree $(1,2)$, i.e. a ruled surface, with the following data: $w_{0,0}=1, w_{0,1}=$ $1, w_{0,2}=2, w_{1,0}=1, w_{1,1}=1, w_{1,2}=2, \mathbf{b}_{0,0}=(1,0,0)$, $\mathbf{b}_{0,1}=(1,0,1), \mathbf{b}_{0,2}=(0,0,1), \mathbf{b}_{1,0}=(1,1,0), \mathbf{b}_{1,1}=$ $(1,1,1), \mathbf{b}_{1,2}=(0,1,0)$. Choosing $\nu=(1,1)$, the matrix $\mathbb{S}_{(1,1)}$ is of size $12 \times 16$ and we find the matrix

$$
\mathbb{M}_{1,1}(\phi)=\left[\begin{array}{cccc}
-1+X & 1-X+Z & 0 & Y \\
-1+X+Z & -2 X & Y & 0 \\
-1+X & 2-2 X+Z & 0 & Y-1 \\
Z & -2 X & Y-1 & 0
\end{array}\right] .
$$

\section{Definition and main properties of implicit matrix representations}

The family of matrices we have built in the previous section is particularly interesting because these matrices can be seen as implicit representations of a parameterized curve or surface, if the degree $\nu$ is not too small. In this section, we describe their main properties. Notice that most of them have been already proved in a more general setting using technics from algebraic geometry and commutative algebra (see $[10,9,6,4])$.

Hereafter, we take again the notation of Section 2: $\phi$ denotes the parameterization (1) of a curve or a surface and $\mathbb{M}_{\nu}(\phi)$ (or $\mathbb{M}_{\nu}$ for short), $\nu \geqslant 0$, its associated family of matrices.

\subsection{Evaluation is linear}

By construction, the entries of the matrices $\mathbb{M}_{\nu}, \nu \geqslant 0$, are linear polynomials in $\mathbb{R}[X, Y, Z]$. Actually, these matrices are pencil of matrices, that is to say that there exist four matrices $M_{\nu, i}, i=0, \ldots, 3$, whose entries are real numbers and such that (see (9))

$$
\mathbb{M}_{\nu}(X, Y, Z)=M_{\nu, 0}+X M_{\nu, 1}+Y M_{\nu, 2}+Z M_{\nu, 3} .
$$


It follows that the matrix $\mathbb{M}_{\nu}=\mathbb{M}_{\nu}(X, Y, Z)$ can be evaluated at any point $P \in \mathbb{R}^{3}$ and that this evaluation is very simple since it corresponds to a linear combination of four matrices. In the sequel, we will denote it by $\mathbb{M}_{\nu}(\phi)(P)$, or simply $\mathbb{M}_{\nu}(P)$ if there is no possible confusion.

\subsection{The drop-of-rank property}

Let $P \in \mathbb{R}^{3}$ be a point such that $P=\phi\left(s_{0}\right)$ for some parameter value $s_{0}$. By construction of $\mathbb{M}_{\nu},(6)$ holds and we hence get

$$
\begin{aligned}
{\left[\psi_{1}\left(s_{0}\right)\right.} & \left.\cdots \psi_{m_{\nu}}\left(s_{0}\right)\right] \times \mathbb{M}_{\nu}(P) \\
& =\left[L_{1}\left(s_{0} ; P\right) \cdots L_{r_{\nu}}\left(s_{0} ; P\right)\right]=\left[\begin{array}{lll}
0 & \cdots & 0
\end{array}\right]
\end{aligned}
$$

Therefore, it is clear that a non trivial linear combination between the rows of $\mathbb{M}_{\nu}$ appears after evaluation at a point $P$ that belongs to the image of $\phi$ (the blending functions $\psi_{i}$ 's do not all vanish simultaneously). It turns out that there exists a "critical degree" $\nu_{0}$ such that, for all matrices $\mathbb{M}_{\nu}$ with $\nu \geqslant \nu_{0}$, the above property characterizes the points $P$ belonging to the (algebraic) closure of the image of $\phi$. For simplicity of presentation, from now on we will denote the image of $\phi$ by $\operatorname{Im}(\phi)$ and its closure by $\overline{\operatorname{Im}}(\phi)$.

To be more precise, we first give explicit values of this critical degree integer $\nu_{0}$ in each of the three cases treated in Section 2:

- $\S 2.1$ : if $\phi$ parameterizes a rational Bézier curve of degree $d \geqslant 1$ then $\nu_{0}:=d-1$.

- $\S 2.2$ : if $\phi$ parameterizes a triangular rational Bézier surface then $\nu_{0}:=2(d-1)$.

- $\S 2.3$ : if $\phi$ parameterizes a tensor-product rational Bézier surface then $\nu_{0}:=\left(2 d_{1}-1, d_{2}-1\right.$ ) (or by symmetry $\left.\nu_{0}=\left(d_{1}-1,2 d_{2}-1\right)\right)$.

Recall that the integers $r_{\nu}$ and $m_{\nu}$ denote the number of columns and rows, respectively, of the matrix $\mathbb{M}_{\nu}$ for all $\nu \geqslant 0(\operatorname{see}(5))$.

Fact 1 For all degrees $\nu \geqslant \nu_{0}$ we have $r_{\nu} \geqslant m_{\nu}$, i.e. the matrices $\mathbb{M}_{\nu}$ have more columns than rows. In particular, $\operatorname{rank}\left(\mathbb{M}_{\nu}(P)\right) \leqslant m_{\nu}$ for all points $P \in \mathbb{R}^{3}$.

Fact 2 For all degrees $\nu \geqslant \nu_{0}$ and all points $P \in \mathbb{R}^{3}$, $\operatorname{rank}\left(\mathbb{M}_{\nu}(P)\right)<m_{\nu}$ if and only if $P \in \overline{\operatorname{Im}}(\phi)$.

The above properties show that for all $\nu \geqslant \nu_{0}$ the matrices $\mathbb{M}_{\nu}(\phi)$ can be used as implicit representations of the parameterization $\phi$. Compared to the more classical implicit representations in the form of polynomial equations in the variables $X, Y, Z$, they are much more easy to compute and they allow us to treat both parameterized curves and surfaces in the same way.

Definition 1 For all $\nu \geqslant \nu_{0}$, the matrices $\mathbb{M}_{\nu}(\phi)$ are called implicit matrix representations (M-rep for short) of the parameterization $\phi$ in degree $\nu$.
The proofs of Fact 1 and Fact 2 can be found in [10], [6] and [4] for the cases of rational curves ( $\$ 2.1)$, triangular (§2.2) and tensor-product (§2.3) rational surfaces respectively. They are independent of any choice of basis (i.e. choices for $\mathcal{B}_{\nu}^{\prime}$ and $\mathcal{B}_{\nu+d}$ ).

Notice that a subtle hypothesis on the parameterization $\phi$ is necessary, namely that the base points (i.e. the common roots of $f_{0}, f_{1}, f_{2}, f_{3}$ in the parameter space, including at infinity) have to be locally defined by at most two equations (what is assumed here for the sake of simplicity). If this is not the case, then one has to deal with some extraneous hyperplanes that appear in addition to $\overline{\operatorname{Im}}(\phi)$, but this is not a problem because these hyperplanes are well understood (see [7] for more details).

Notice also that the values given above for the critical degree $\nu_{0}$ can be improved in some cases. For instance, for rational curves $\nu_{0}$ can be decreased by the smallest degree $\nu$ such that there exists a non trivial 4-tuple $\left(g_{0}, g_{1}, g_{2}, g_{3}\right)$ satisfying (2), so at least by 1 if the curve is not a line (see [10]). For the case of triangular rational surfaces, $\nu_{0}$ can be decreased by the smallest degree of a curve in the parameter space going through all the base points, which is at least 1 if there exist base points (see [6]). However, all these improved values depend on particular geometric features of the parameterization $\phi$ and are hence not stable under numerical perturbations. This is why we emphasized the smallest values of the critical degree that only depend on the degree of the $f_{i}$ 's and that are hence numerically stable.

Example 4 Coming back to the examples given in Section 2, we see that $\mathbb{M}_{\nu}$ is an $M$-rep for all $\nu \geqslant \nu_{0}=2$ in Example 1 , but $\mathbb{M}_{1}$ is actually also an $M$-rep because this curve is not a line.

In Example 2, $\mathbb{M}_{\nu}$ is an $M$-rep of the sphere for all $\nu \geqslant$ $\nu_{0}=2$, but $\mathbb{M}_{1}$ is also an $M$-rep because the two cyclic points are base points.

In Example 3, the matrix $\mathbb{M}_{\left(\nu_{1}, \nu_{2}\right)}$ is an $M$-rep for all $\left(\nu_{1}, \nu_{2}\right) \geqslant(1,1)($ or $\geqslant(0,3))$. Notice that $\mathbb{M}_{(1,1)}$ is a square matrix, so a polynomial implicit equation of the ruled surface can be obtained by computing its determinant. A similar property holds for $\mathbb{M}_{0,3}$.

Finally, although we choose to work in the affine space $\mathbb{R}^{3}$, there is no hidden difficulty at infinity. Indeed, denoting by $(W: X: Y: Z)$ the homogeneous coordinates of the projective space $\mathbb{P}^{3}(\mathbb{R})$ (with the convention $(1: X: Y$ : $Z)=(X, Y, Z)$ in $\left.\mathbb{R}^{3}\right)$, the pencil of matrices

$$
W M_{\nu, 0}+X M_{\nu, 1}+Y M_{\nu, 2}+Z M_{\nu, 3}
$$

have the properties stated above in $\mathbb{P}^{3}(\mathbb{R})$ and hence it provides an implicit matrix representation of the closure of the image of $\phi$ in $\mathbb{P}^{3}(\mathbb{R})$. Actually, one can even replace the field of real numbers by any other field (including the field of complex numbers) and the above properties, as well as the construction of $\mathbb{M}_{\nu}$, still hold. 


\subsection{The inversion property}

Let $P \in \mathbb{R}^{3}$ be a point such that $P=\phi\left(s_{0}\right)$ for some parameter value $s_{0}$. For any M-rep $\mathbb{M}_{\nu}$ of the parameterization $\phi$, we have seen that the equality (12) holds so that the vector

$$
\left[\psi_{1}\left(s_{0}\right) \cdots \psi_{m_{\nu}}\left(s_{0}\right)\right]^{T}
$$

belongs to the null space of $\mathbb{M}_{\nu}(P)^{T}$ (the notation $-{ }^{T}$ stands for the matrix transpose).

Fact 3 If $P \in \mathbb{R}^{3}$ is a point such that $P$ has a unique (counted properly) pre-image by $\phi$, then the dimension of the null space of $\mathbb{M}_{\nu}(P)^{T}$ is one.

This property shows that an M-rep allows us to invert a point $P=\phi\left(s_{0}\right)$ on the curve or surface parameterized by $\phi$ if $\phi$ is proper and $P$ does not belong to the self-intersection locus (notice that if $\phi$ is not proper or if $P$ belongs to the self-intersection locus, then inversion is not well-defined). Indeed, the computation of the null space of $\mathbb{M}_{\nu}(P)^{T}$ provides a single vector $\left[\begin{array}{llll}v_{1} & \cdots & v_{m_{\nu}}\end{array}\right]^{T}$ which is proportional to the vector (13). From here, the parameter $s_{0}$ can be extracted without any difficulty. For instance, if $\phi$ parameterizes a rational Bézier curve (\$2.1), then looking at the ratio (but other ratios can be used as well)

$$
\left(v_{1}: v_{2}\right)=\left(B_{0}^{d}\left(s_{0}\right): B_{1}^{d}\left(s_{0}\right)\right)=\left(1-s_{0}: d s_{0}\right)
$$

we deduce that $s_{0}=v_{2} /\left(d v_{1}+v_{2}\right)$ (notice that the denominator can never vanish). If $\phi$ parameterizes a triangular (§2.2) or a tensor-product (§2.3) rational Bézier surface, we can proceed similarly and the computation of two ratios yields the two coordinates of the pre-image of $P$.

With an M-rep, it is hence possible to handle at the same time a non-singular point $P \in \operatorname{Im}(\phi)$ and its pre-image through a simple null space computation. This property can be very useful in many circumstances, as for instance for splitting a curve at a point in space, or for dealing with trimmed surfaces.

To simplify our notation, in the sequel we will denote by the corank of a given matrix $M$ the difference between its number of rows and its rank. Equivalently, $\operatorname{corank}(M)$ is equal to the dimension of the left null space of $M$.

\subsection{Pre-images and the drop-of-rank property}

Let $\mathbb{M}_{\nu}$ be an M-rep of a parameterization $\phi$ and let $P$ be a point in $\mathbb{R}^{3}$. We have seen (see Fact 1 and Fact 2 ) that $\operatorname{corank}\left(\mathbb{M}_{\nu}(P)\right)=0$ if and only if $P \notin \overline{\operatorname{Im}}(\phi)$. Moreover, in Fact 3 we stated that $\operatorname{corank}\left(\mathbb{M}_{\nu}(P)\right)=1$ if $P$ is a nonsingular point of the closure of the image of $\phi$. It turns out that some even finer geometric properties of $\phi$ can be extracted from an M-rep. For the sake of completeness, we briefly mention them.

Denote by $\phi^{-1}(P)$ the algebraic set of all the pre-images of $P$ by $\phi$ in the parameter space over the field of complex numbers. Thus, if $P \notin \overline{\operatorname{Im}}(\phi)$ then $\phi^{-1}(P)$ is empty. Oth- erwise, $\phi^{-1}(P)$ can be either a finite set or an infinite set of points. If $\phi^{-1}(P)$ is finite, which is for instance always the case if $\phi$ parameterizes a rational curve, then we denote by $N_{P}$ its cardinal number, counted properly with multiplicities. If $\phi^{-1}(P)$ is infinite, which can only occur if $\phi$ parameterizes a surface, then it can be decomposed into the union of an algebraic plane curve and a finite set of points; we denote by $D_{P}$ the degree of the curve.

The proof of the following result is beyond the scope of this paper and can be found in [5].

Fact 4 Let $P$ be a point in $\mathbb{R}^{3}$ and $\mathbb{M}_{\nu}$ be an $M$-rep of $\phi$.

- If $\phi^{-1}(P)$ is finite, then for all $\nu \geqslant \nu_{0}$

$$
\operatorname{corank}\left(\mathbb{M}_{\nu}(P)\right)=N_{P}
$$

- If $\phi^{-1}(P)$ is infinite, then for all $\nu \geqslant \nu_{0}$

$$
\operatorname{corank}\left(\mathbb{M}_{\nu}(P)\right)=D_{P} \cdot \nu+C_{P}
$$

Two comments are in order about this fact. First, if $\phi$ parameterizes tensor-product rational Bézier surface $(\S 2.3)$, $D_{P}$ is a bi-degree, say $D_{P}=\left(D_{1}, D_{2}\right)$, and $D_{P} . \nu$ is the "scalar product" $D_{1} \cdot \nu_{1}+D_{2} \cdot \nu_{2}$ where $\nu=\left(\nu_{1}, \nu_{2}\right)$. Second, mention that the quantity $C_{P}$ has a geometric meaning. It gathers informations about the genus of the curve component of $\phi^{-1}(P)$ and the cardinal number of the remaining finite part, counted properly with multiplicity. In algebraic geometry, the linear polynomial $D_{P} . \nu+C_{P}$ is actually known as the Hilbert polynomial of $\phi^{-1}(P)$ (see e.g. [14, Chapter 6, §4]).

A direct consequence of Fact 4 is that the comparison of the corank of two or three successive M-reps $\left(\mathbb{M}_{\nu}\right.$ and $\mathbb{M}_{\nu+1}$ for a triangular surface and $\mathbb{M}_{\nu}, \mathbb{M}_{\nu+(0,1)}, \mathbb{M}_{\nu+(1,0)}$ for a tensor-product surface) allows us to determine if $\phi^{-1}(\phi)$ is finite or infinite and at the same time to determine $N_{P}$ or $D_{P}$. Another interesting observation is that small drop of rank can only arises at points $P$ such that $\phi^{-1}(P)$ is finite. We refer the reader to [5] for more details.

\section{Evaluating M-reps through singular values}

In this section, we will define a real evaluation function of an M-rep $\mathbb{M}_{\nu}$ at a given point $P$ by means of the singular values of the matrix $\mathbb{M}_{\nu}(P)$. For any point $P \in \mathbb{R}^{3}$, we will hence get a real number denoted $\delta_{\mathbb{M}_{\nu}}(P) \in \mathbb{R}$. We will show that this real evaluation function behaves like a classical Euclidean distance function. As a byproduct, we will also get a new determinantal formula for implicitizing a curve or surface over the real numbers. We begin with a quick review of the SVD.

\subsection{The singular value decomposition}

Let $A \in \mathbb{R}^{m \times r}$ be a real matrix and assume for ease of presentation that $m \leqslant r$ (if $m>r$ then simply apply what follows to $A^{T}$ ). The singular value decomposition (SVD) of $A$ is a decomposition of the form $A=U \Sigma V^{T}$ where $U=$ 
$\left[u_{1}, \ldots, u_{m}\right] \in \mathbb{R}^{m \times m}$ and $V=\left[v_{1}, \ldots, v_{r}\right] \in \mathbb{R}^{r \times r}$ are real orthogonal matrices and the matrix

$$
\Sigma=\left[\begin{array}{ccccccc}
\sigma_{1} & 0 & \ldots & 0 & 0 & \ldots & 0 \\
0 & \sigma_{2} & \ddots & \vdots & \vdots & & \vdots \\
\vdots & \ddots & \ddots & 0 & 0 & \ldots & 0 \\
0 & \ldots & 0 & \sigma_{m} & 0 & \ldots & 0
\end{array}\right] \in \mathbb{R}^{m \times r}
$$

has nonnegative diagonal elements appearing in the conventional decreasing order

$$
\sigma_{1} \geqslant \sigma_{2} \geqslant \cdots \geqslant \sigma_{m} \geqslant 0
$$

The numbers $\sigma_{i}$, also denoted $\sigma_{i}(A)$, are called the singular values of $A$ while the vectors $u_{i}$ and $v_{i}$ are called the left and right singular vectors of $A$, respectively. Notice that the singular values of $A$ are precisely the lengths of the semi-axes of the ellipsoidal image of the unit sphere under the mapping $x \rightarrow A x$. We refer the reader to $[17, \S 2.5 .3]$ for the proof of the existence of the SVD.

The most important property of the SVD for our purposes is that it is a powerful tool for deciding the rank of a matrix. We will come back to this in the next section when dealing with numerical computations. For now, we will only need the following elementary property:

$$
\operatorname{rank}(A)=\max \left\{i: \sigma_{i}(A) \neq 0\right\} .
$$

\subsection{Real evaluation function of an $M$-rep}

Consider a parameterization $\phi$ of a curve or surface as in (1) and let $\mathbb{M}_{\nu}$ be an M-rep of $\phi$. Recall from Section 2 that the matrix $\mathbb{M}_{\nu}$ has $m_{\nu}$ rows and $r_{\nu}$ columns.

Definition 2 For any point $P$ in $\mathbb{R}^{3}$, the real evaluation function of $\mathbb{M}_{\nu}$ at $P$ is defined by

$$
\begin{aligned}
& \delta_{\mathbb{M}_{\nu}}: \mathbb{R}^{3} \rightarrow \mathbb{R}_{\geqslant 0}:=\{x \in \mathbb{R}: x \geqslant 0\} \\
& P \mapsto \delta_{\mathbb{M}_{\nu}}(P):=\prod_{i=1}^{m_{\nu}} \sigma_{i}\left(\mathbb{M}_{\nu}(P)\right)
\end{aligned}
$$

where $\sigma_{i}\left(\mathbb{M}_{\nu}(P)\right)$ are the singular values of the matrix $\mathbb{M}_{\nu}(P) \in \mathbb{R}^{m_{\nu} \times r_{\nu}}$.

For any point $P, \delta_{\mathbb{M}_{\nu}}(P) \geqslant 0$ and Fact 2 and (14) imply that

$$
\delta_{\mathbb{M}_{\nu}}(P)=0 \Leftrightarrow P \in \overline{\operatorname{Im}}(\phi) .
$$

Therefore, the function $\delta_{\mathbb{M}_{\nu}}(P)$ can be seen as a sort of distance function of the point $P$ to $\overline{\operatorname{Im}}(\phi)$ (i.e. the curve or surface parameterized by $\phi)$. This is similar to the algebraic distance function obtained by returning the absolute value of the evaluation of an implicit polynomial equation $F(X, Y, Z)=0$ of a parameterized surface: $|F(P)|=0$ if and only if $P$ belongs to the surface.

It turns out that the evaluation function $\delta_{\mathbb{M}_{\nu}}(P)$ can actually be compared with the usual Euclidean distance function of $P$ to $\overline{\operatorname{Im}}(\phi) \subset \mathbb{R}^{3}$. To start, we first show that the square of $\delta_{\mathbb{M}_{\nu}}$ is an algebraic function and point out an interesting consequence.
4.3. A real implicit polynomial equation of parameterized curves and surfaces

Let $\mathbb{M}_{\nu}$ be an M-rep of the parameterization $\phi$ and $P$ a point in $\mathbb{R}^{3}$. From the SVD of $\mathbb{M}_{\nu}$ and the definition of $\delta_{\mathbb{M}_{\nu}}(P)$, we get

$$
\delta_{\mathbb{M}_{\nu}}(P)^{2}=\prod_{i=1}^{m_{\nu}} \sigma_{i}\left(\mathbb{M}_{\nu}(P)\right)^{2}=\operatorname{det}\left(\mathbb{M}_{\nu}(P) \mathbb{M}_{\nu}(P)^{T}\right) .
$$

Since the entries of $\mathbb{M}_{\nu}$ are linear polynomials in $\mathbb{R}[X, Y, Z]$, we deduce that $\delta_{\mathbb{M}_{\nu}}(P)^{2}$ is a polynomial function of $P$. Therefore, we define the polynomial

$$
\Delta_{\mathbb{M}_{\nu}}(X, Y, Z):=\operatorname{det}\left(\mathbb{M}_{\nu} \mathbb{M}_{\nu}^{T}\right) \in \mathbb{R}[X, Y, Z] .
$$

For any $P \in \mathbb{R}^{3}$ we have $\Delta_{\mathbb{M}_{\nu}}(P)=\delta_{\mathbb{M}_{\nu}}(P)^{2}$.

Theorem 1 The real algebraic set

$$
\left\{(x, y, z) \in \mathbb{R}^{3}: \Delta_{\mathbb{M}_{\nu}}(x, y, z)=0\right\} \subset \mathbb{R}^{3}
$$

is equal to $\overline{\operatorname{Im}}(\phi)$. In other words, $\Delta_{\mathbb{M}_{\nu}}(X, Y, Z)$ is a real implicit equation of the curve or surface parameterized by $\phi$.

Proof. It is a direct consequence of the definition of the polynomial $\Delta_{\mathbb{M}_{\nu}}$ and (15). Another way to see this result is to apply the Binet-Cauchy formula for expanding the determinant of the product $\mathbb{M}_{\nu} \mathbb{M}_{\nu}^{T}$. Indeed, this formula yields

$$
\operatorname{det}\left(\mathbb{M}_{\nu} \mathbb{M}_{\nu}^{T}\right)=\sum_{1 \leqslant i_{1}<i_{2}<\cdots<i_{m_{\nu}} \leqslant r_{\nu}}\left(\left[\mathbb{M}_{\nu}\right]_{i_{1}, i_{2}, \ldots, i_{m_{\nu}}}\right)^{2}
$$

where $\left[\mathbb{M}_{\nu}\right]_{i_{1}, i_{2}, \ldots, i_{m_{\nu}}}(X, Y, Z)$ is the minor of $\mathbb{M}_{\nu}$ corresponding to the columns $i_{1}, i_{2}, \ldots, i_{m_{\nu}}$. Then, the conclusion follows from Fact 2.

The matrix $\mathbb{M}_{\nu} \mathbb{M}_{\nu}^{T}$ is a symmetric square matrix of size $m_{\nu}$ and its entries are quadratic polynomials in $\mathbb{R}[X, Y, Z]$. Its determinant $\Delta_{\mathbb{M}_{\nu}}$ is hence a degree $2 m$ polynomial and it is moreover a sum of squares by (16). Looking more precisely at the three cases detailed in Section 2 with $\nu=\nu_{0}$, we get:

- §2.1: if $\phi$ parameterizes a rational Bézier curve of degree $d$ then $m_{\nu_{0}}=\nu_{0}+1=d, \mathbb{M}_{\nu} \mathbb{M}_{\nu}^{T}$ is of size $d$ and $\Delta_{\mathbb{M}_{\nu}}$ is a polynomial of degree $2 d$.

- $\oint_{2.2:}$ if $\phi$ parameterizes a triangular rational Bézier surface of degree $d$ then $m_{\nu_{0}}=d(2 d-1)$ and $\Delta_{\mathbb{M}_{\nu}}$ is a polynomial of degree $2 d(2 d-1)$.

- $\S 2.3$ : if $\phi$ parameterizes a tensor-product rational Bézier surface of bi-degree $\left(d_{1}, d_{2}\right)$ then $m_{\nu_{0}}=2 d_{1} d_{2}$ and $\Delta_{\mathbb{M}_{\nu}}$ is a polynomial of degree $4 d_{1} d_{2}$.

Theorem 1 shows that implicit matrix representations allow us to produce square matrices whose determinant is an implicit polynomial equation for a parameterized curve or surface over the real numbers. In general, this equation is not of the lowest possible degree, see e.g. Example 5. Notice too that Theorem 1 can be extended to the field of complex numbers by taking the Hermitian matrix transpose instead 
of the simple matrix transpose (the Hermitian transpose of a complex matrix is obtained by taking its transpose and then by taking the complex conjugate of each entry).

Example 5 Taking again Example 2, the computation of the product $\mathbb{M}_{1} \mathbb{M}_{1}^{T}$ yields a $3 \times 3$-matrix which is symmetric and has quadratic entries in $\mathbb{R}[X, Y, Z]$. Its determinant $\Delta_{\mathbb{M}_{1}}(X, Y, Z)$ is equal to

$$
\left((X-Y+1)^{2}+2 Y^{2}+Z^{2}\right) \times\left(X^{2}+Y^{2}+Z^{2}-1\right)^{2} .
$$

The right factor is the usual implicit equation of the sphere. The left factor vanishes over the real numbers only at the point $(X, Y, Z)=(-1,0,0)$ which belongs to the sphere.

\subsection{Comparison with the Euclidean distance function}

In the field of real algebraic geometry, the Eojasiewicz inequality is a classical result that gives information concerning the relative rate of growth of two continuous semialgebraic functions (see for instance [3]). In our context, it can be used to compare the growth of the evaluation function given in Definition 2 with the one of the usual Euclidean distance between $P$ and $\overline{\operatorname{Im}}(\phi)$. This latter will be denoted by

$$
d(P, \overline{\operatorname{Im}}(\phi)):=\min \left\{\|P-Q\|_{2}: Q \in \overline{\operatorname{Im}}(\phi)\right\} .
$$

Theorem 2 Let $K \subset \mathbb{R}^{3}$ be a compact semialgebraic set. Then there exist two positive integers $n_{1}, n_{2}$ and two positive real numbers $c_{1}, c_{2}$ such that

$$
\forall P \in K \quad d(P, \overline{\operatorname{Im}}(\phi))^{n_{1}} \leqslant c_{1} \cdot \delta_{\mathbb{M}_{\nu}}(P)
$$

and

$$
\forall P \in K \quad \delta_{\mathbb{M}_{\nu}}(P)^{n_{2}} \leqslant c_{2} \cdot d(P, \overline{\operatorname{Im}}(\phi)) .
$$

Proof. Both functions $\delta_{\mathbb{M}_{\nu}}$ and $d(-, \overline{\operatorname{Im}}(\phi))$ are continuous, and they are also semialgebraic as they are the square roots of positive algebraic functions. Moreover, the zero locus of these two functions are equal:

$$
\begin{aligned}
& \left\{P \in \mathbb{R}^{3}: d(P, \overline{\operatorname{Im}}(\phi))=0\right\} \\
& \quad=\left\{P \in \mathbb{R}^{3}: \delta_{\mathbb{M}_{\nu}}(P)=0\right\}=\overline{\operatorname{Im}}(\phi) .
\end{aligned}
$$

It follows that, after restriction to the compact semialgebraic set $K$, all the hypothesis for applying Eojasiewicz inequality are fulfilled (see $[3, \S 6]$ ) and we thus get the two inequalities stated in this theorem.

This result shows that the evaluation function $\delta_{\mathbb{M}_{\nu}}$ behaves similar to a distance function: its value increases as one gets far from $\overline{\operatorname{Im}}(\phi)$ and decreases as one gets close to it until vanishing exactly on it. The compact semi-algebraic set $K$ can be taken as a 3D bounding-box, but notice that there exists a version of Łojasiewicz inequality where $K$ is not assumed to be a compact set (for instance $K$ could be $\mathbb{R}^{3}$ ). Finally, notice that determining the integers $n_{1}, n_{2}$ and constants $c_{1}, c_{2}$ is in general difficult, although some effective bounds are known. We refer the reader to [26] (and the references therein; see also [21]) for more details.

\section{Numerical behavior of M-reps}

Implicit matrix representations are interesting not only for their nice geometric properties, but also because all the experiments we have conducted have shown a particularly good numerical stability. One can expect that this is a consequence of the design of M-reps that makes them very well adapted to the SVD which is one of the basic and most important tool of numerical linear algebra. Indeed, as we will explain in this section, all the properties of M-reps can be exploited using numerical computations by means of the SVD, even their construction. In addition, we provide some rigorous results on the properties of M-reps under numerical computations in order to give more insight on the good numerical behavior of M-reps we have observed in our experiments.

\subsection{The numerical rank}

In the presence of roundoff errors and data perturbations, every matrix tends to be a full rank matrix and hence determining its original rank becomes nontrivial. A strict and operational definition of "numerical rank", which is widely used in the field of numerical linear algebra, takes the following form.

Definition 3 ([17, §2.5.5]) The numerical rank of a matrix $A$, with respect to the tolerance $\epsilon>0$, is given by

$$
\operatorname{rank}(A, \epsilon)=\min \left\{\operatorname{rank}(B):\|A-B\|_{2} \leqslant \epsilon\right\} .
$$

In other words, the numerical rank of $A$ is equal to the number of columns of $A$ that are guaranteed to be linearly independent for any perturbation of $A$ with 2-norm less than or equal to the tolerance $\epsilon$.

An important property of the SVD is that for all nonnegative integers $k<\operatorname{rank}(A)$,

$$
\sigma_{k+1}(A)=\min _{\operatorname{rank}(B)=k}\|A-B\|_{2}
$$

which means that the singular value $\sigma_{k+1}(A)$ indicates how near the matrix $A$ is to a matrix of rank $k$ (see [17, Theorem 2.5.3]). Therefore, setting $r_{\epsilon}:=\operatorname{rank}(A, \epsilon)$, we see that

$$
\sigma_{1}(A) \geqslant \cdots \geqslant \sigma_{r_{\epsilon}}(A)>\epsilon \geqslant \sigma_{r_{\epsilon+1}}(A) \geqslant \cdots \geqslant \sigma_{m}(A)
$$

with the notation $A \in \mathbb{R}^{m \times r}, m \leqslant r$. The numerical rank of $A$ can hence be determined by inspecting the singular values of $A$ :

$$
\operatorname{rank}(A, \epsilon):=\max \left\{k: \sigma_{k}(A)>\epsilon\right\} .
$$

Due to computer arithmetic, the calculated singular values may be different from the exact singular values. However, on can show (see $[17, \S 5.5 .8]$ ) that the computed singular values of $A$ are the exact singular values of a slightly perturbed matrix $A+E$ where $\|E\|_{2} \leqslant \rho u\|A\|_{2}$; here $\mathbf{u}$ is the roundoff unit (see $[17, \S 2.4 .2]$ ) and $\rho$ is a slowly growing function of $m$ and $r$. Therefore, in this context the tolerance $\epsilon$ is usually chosen in this form, so that it makes sense 
in (18) to use the computed singular values of $A$ in place of the exact singular values of $A$.

\subsection{Numerical computation of $M$-reps}

Let $\phi$ be a parameterization as in (1). As described in Section 2, an M-rep $\mathbb{M}_{\nu}$ of $\phi$ is obtained as the null space of the multiplication matrix $\mathbb{S}_{\nu}$ which is built directly from the control points and weights of $\phi$ (see e.g. (8)). We will describe how this computation can be done numerically by means of the SVD.

Consider a matrix $A$ and its SVD $A=U \Sigma V^{T} \in \mathbb{R}^{m \times r}$ where $V=\left[v_{1}, \ldots, v_{r}\right] \in \mathbb{R}^{r \times r}$. It is easy to check that

$$
\left\|A v_{i}\right\|_{2}=\sigma_{i}(A), \quad i=1, \ldots, r
$$

(setting $\sigma_{i}(A)=0$ if $i>\max (m, r)$ ). So, if $\sigma_{i}(A)$ is "small" compared to the norm $\|A\|_{2}=\sigma_{1}(A)$, then the corresponding right singular vector $v_{i}$ is "almost" a null vector for $A$. It follows that the determination of the numerical rank $r_{\epsilon}$ of $A$, with the tolerance $\epsilon$, yields a numerical null space of $A$ which is the vector space spanned by the vectors $v_{r_{\epsilon}+1}, \ldots, v_{r}$.

From these considerations, we deduce that $\mathbb{M}_{\nu}$ can be read off the SVD of the matrix $\mathbb{S}_{\nu}$. Indeed, with the appropriate tolerance $\epsilon$, this SVD yields a numerical rank $r_{\epsilon}$ and the last $r-r_{\epsilon}$ columns in the matrix $V$ yield the numerical null space of $\mathbb{S}_{\nu}$. It is of the form (8) and hence provides a numerical approximation of $\mathbb{M}_{\nu}$ given by

$$
M_{\nu, 0}+X M_{\nu, 1}+Y M_{\nu, 2}+Z M_{\nu, 3} .
$$

The quality of this numerical approximation is essentially governed by the ratio $\sigma_{1} / \sigma_{r_{\epsilon}}$. To be more precise, for a given matrix $A$, denote by $\mathcal{N}_{k}(A)$ the vector space spanned by the vectors $v_{k+1}, \ldots, v_{r}$. Let $A+E$ be the small perturbation of the matrix $A$ corresponding to the computation of its singular values (see $\S 5.1$ ), so that the computed singular values of $A$ are the exact singular values of $A+E$. It can be shown ([18, Theorem 3.2.1], see also [17, §8.6.1]) that if $\|E\|_{2} \leqslant \sigma_{k}-\sigma_{k+1}$, then

$$
\operatorname{dist}\left(\mathcal{N}_{k}(A), \mathcal{N}_{k}(A+E)\right) \leqslant \frac{\sigma_{1}}{\sigma_{k}} \cdot \frac{\|E\|_{2}}{\|A\|_{2}}
$$

where dist $(-,-)$ stands for the distance between vector spaces (see $[17, \S 2.6 .3]$ ). In our setting, we deduce that the SVD of $\mathbb{S}_{\nu}$ will give an accurate and robust numerical null space (hence numerical M-rep $\mathbb{M}_{\nu}$ ), namely $\mathcal{N}_{r-r_{\epsilon}+1}\left(\mathbb{S}_{\nu}\right)$, if there is a distinct gap between $\sigma_{r_{\epsilon}}$ and $\sigma_{r_{\epsilon}+1}$ with respect to $\sigma_{1}$

Example 6 Taking again Example 2, the computation of the SVD of the $10 \times 12$ multiplication matrix $\mathbb{S}_{1}$ returns

$$
\begin{gathered}
\sigma_{1}=3.52756346141076, \sigma_{8}=0.452628072697747, \\
\sigma_{9}=3.31295025717184461 \times 10^{-11} .
\end{gathered}
$$

We deduce that the numerical rank is equal to 8 and hence find a null space of dimension 4. The numerical M-rep $\mathbb{M}_{1}(\phi)$ we get this way is printed in Figure 1.
The numerical computation of M-reps have some similarities with approximate implicitization [16,2], as the use of the SVD, but it is different. In particular, with M-reps there is no need to guess or estimate a good degree for the approximate implicit representation, it is provided by the method: this is the integer $\nu_{0}$ (see $\left.\S 3.2\right)$. Its determination is actually the difficult part of the M-rep approach for which tools from commutative algebra and algebraic geometry are necessary.

\subsection{The drop-of-rank property and numerical computations}

Let $\mathbb{M}_{\nu}$ be an M-rep of a parameterization $\phi$ as in (1), and let $P$ be a point in $\mathbb{R}^{3}$. The drop-of-rank property of $\mathbb{M}_{\nu}$ is the fact that the rank of $\mathbb{M}_{\nu}$ drops at $P$ if and only if $P \in \overline{\operatorname{Im}}(\phi)$. Therefore, it seems quite natural to compute the SVD of $\mathbb{M}_{\nu}(P)$ for deciding if $P$ belongs to $\overline{\operatorname{Im}}(\phi)$. However, one has to be careful because there is a subtle difficulty. Indeed, the theory of the SVD have been shown to be very powerful when dealing with the whole space of dense matrices. For instance, looking at (17) it is clear that the matrix $B$ can be any matrix without particular structure, even if $A$ has such a particular structure itself. In our setting, we are handling M-reps which are pencils of matrices (see (9)) and hence are very particular. Therefore, there is no guarantee that the detection of a numerical drop of rank at a given point $Q$ corresponds to the existence of another point $P$ in its neighborhood such that the exact rank of $\mathbb{M}_{\nu}(P)$ drops. This is because M-reps have this particular structure of being a pencil of matrices. In general, working with such particular subspaces of matrices may lead to difficulties with the SVD. However, all the experiments we have made with M-reps have always shown a very good numerical behavior. In order to explain and clarify these observations, we prove two results showing that a given point $P$ is close to $\overline{\operatorname{Im}}(\phi)$ if and only if the numerical rank of $\mathbb{M}_{\nu}$ drops at $P$. In the sequel, it is assumed that M-reps are computed as explained in $\S 5.2$. Recall also the notation that an M-rep $\mathbb{M}_{\nu}$ is of size $m_{\nu} \times r_{\nu}$.

Proposition 1 Let $P, Q$ be two points in $\mathbb{R}^{3}$. For all $k=$ $1, \ldots, m_{\nu}$, we have

$$
\left|\sigma_{i}\left(\mathbb{M}_{\nu}(P)\right)-\sigma_{i}\left(\mathbb{M}_{\nu}(Q)\right)\right| \leqslant\|P-Q\|_{2} .
$$

Proof. We begin with a useful remark: the matrix $\mathbb{M}_{\nu}(P)$ can be computed as described in $\S 3.1$, but it is not hard to check that it can also be computed as the product of two matrices. Indeed, to any point $P=(x, y, z) \in \mathbb{R}^{3}$ we associate the following matrix

$$
\mathbb{P}_{\nu}=\left[\begin{array}{c}
\operatorname{diag}(1) \\
\operatorname{diag}(x) \\
\operatorname{diag}(y) \\
\operatorname{diag}(z)
\end{array}\right]
$$

where each block is a diagonal matrix of size $m_{\nu}$ with the same repeated element on the diagonal (from top to bot- 
$\left[\begin{array}{cccc}-0.000+0.000 X-0.354 Y+0.354 Z & 0.0811-0.0811 X+0.324 Y+0.324 Z & -0.354+0.354 X+0.000 Y-0.000 Z & -0.005+0.005 X-0.000 Y+0.000 Z \\ -0.354-0.354 X+0.000 Y+0.354 Z & -0.243-0.406 X+0.324 Y+0.243 Z & -0.354+0.354 X+0.0107 Y+0.354 Z-0.005+0.005 X-0.707 Y+0.005 Z \\ 0.354+0.354 X-0.354 Y+0.000 Z & -0.243-0.406 X+0.243 Y+0.324 Z & -0.354+0.354 X+0.354 Y-0.0107 Z-0.005+0.005 X+0.005 Y+0.707 Z\end{array}\right]$

Fig. 1. Numerical M-rep for the unit sphere with a display precision of three digits.

tom, the diagonal of the first block is filled with 1 , the diagonal of the second block with $\mathrm{x}$, and so on). Then, denoting by $\mathbb{N}_{\nu}$ the null space of $\mathbb{S}_{\nu}$ (see (8)), we get that

$$
\mathbb{M}_{\nu}(P)=\mathbb{N}_{\nu}^{T} \mathbb{P}_{\nu}
$$

Now, by applying a classical inequality about singular values (see [17, Corollary 8.6.2]), we get

$$
\left|\sigma_{i}\left(\mathbb{M}_{\nu}(P)\right)-\sigma_{i}\left(\mathbb{M}_{\nu}(Q)\right)\right| \leqslant\left\|\mathbb{M}_{\nu}(P)-\mathbb{M}_{\nu}(Q)\right\|_{2} .
$$

But using (20) and a standard inequality for the 2-norm of matrices, we obtain

$$
\begin{aligned}
\left\|\mathbb{M}_{\nu}(P)-\mathbb{M}_{\nu}(Q)\right\|_{2} & =\left\|\mathbb{N}_{\nu}^{T}\left(\mathbb{P}_{\nu}-\mathbb{Q}_{\nu}\right)\right\|_{2} \\
& \leqslant\left\|\mathbb{N}_{\nu}^{T}\right\|_{2}\left\|\mathbb{P}_{\nu}-\mathbb{Q}_{\nu}\right\|_{2}
\end{aligned}
$$

The matrix $\mathbb{P}_{\nu}-\mathbb{Q}_{\nu}$ is made of columns that are orthogonal vectors whose norm is equal to $\|P-Q\|_{2}$, so

$$
\left\|\mathbb{P}_{\nu}-\mathbb{Q}_{\nu}\right\|_{2}=\|P-Q\|_{2} .
$$

The matrix $\mathbb{N}_{\nu}$ is made of orthonormal columns (see $\S 5.2$ ), so $\left\|\mathbb{N}_{\nu}^{T}\right\|_{2}=\left\|\mathbb{N}_{\nu}\right\|_{2}=1$ and the conclusion follows.

Corollary 1 Let $P$ be a point in $\mathbb{R}^{3}$ and $\epsilon>0$ be a tolerance. For all points $P_{\epsilon} \in \mathbb{R}^{3}$ such that $\left\|P_{\epsilon}-P\right\|_{2} \leqslant \epsilon$ we have

$$
\operatorname{rank}\left(\mathbb{M}_{\nu}\left(P_{\epsilon}\right), \epsilon\right) \leqslant \operatorname{rank}\left(\mathbb{M}_{\nu}(P)\right) .
$$

Proof. For all integers $k>\operatorname{rank}\left(\mathbb{M}_{\nu}(P)\right), \sigma_{k}\left(\mathbb{M}_{\nu}(P)\right)=0$ and the conclusion follows by applying Proposition 1.

This result shows that if a point $P$ is close to $\overline{\operatorname{Im}}(\phi)$ then the numerical rank of $\mathbb{M}_{\nu}$ drops at $P$. Now, we examine the converse.

Proposition 2 Let $P$ be a point in compact semialgebraic set $K \subset \mathbb{R}^{3}, \epsilon>0$ be a tolerance and set $r_{\epsilon}:=$ $\operatorname{rank}\left(\mathbb{M}_{\nu}(P), \epsilon\right), \sigma_{1}:=\sigma_{1}\left(\mathbb{M}_{\nu}(P)\right)$. There exist a positive integer $n_{1}$ and a constant real number $c_{1}$ such that

$$
d(P, \overline{\operatorname{Im}}(\phi)) \leqslant\left(c_{1} \sigma_{1}^{r_{\epsilon}} \epsilon^{m_{\nu}-r_{\epsilon}}\right)^{\frac{1}{n_{1}}} .
$$

Moreover, $\sigma_{1} \leqslant\left(1+\|P\|_{2}^{2}\right)^{\frac{1}{2}}$.

Proof. By Theorem 2, there exist a positive integer $n_{1}$ and a constant real number $c_{1}$ such that for all points $P \in K$

$$
d(P, \overline{\operatorname{Im}}(\phi))^{n_{1}} \leqslant c_{1} \delta_{\mathbb{M}_{\nu}(P)}(P)=c_{1} \prod_{k=1}^{m_{\nu}} \sigma_{k}\left(\mathbb{M}_{\nu}(P)\right) .
$$

Now, by the property of the numerical rank, the singular values of $\mathbb{M}_{\nu}(P)$ satisfy the inequalities

$$
\sigma_{1} \geqslant \cdots \geqslant \sigma_{r_{\epsilon}}>\epsilon \geqslant \sigma_{r_{\epsilon+1}} \geqslant \cdots \geqslant \sigma_{m_{\nu}} .
$$

Therefore, we deduce that

$$
\prod_{k=1}^{m_{\nu}} \sigma_{k}\left(\mathbb{M}_{\nu}(P)\right) \leqslant \sigma_{1}^{r_{\epsilon}} \epsilon^{m_{\nu}-r_{\epsilon}}
$$

and hence

$$
d(P, \overline{\operatorname{Im}}(\phi))^{n_{1}} \leqslant c_{1} \sigma_{1}^{r_{\epsilon}} \epsilon^{m_{\nu}-r_{\epsilon}} .
$$

To prove the second claimed inequality, we observe that $\sigma_{1}=\left\|\mathbb{N}_{\nu}^{T} \mathbb{P}_{\nu}\right\|_{2}$ by (20). It follows that $\sigma_{1} \leqslant\left\|\mathbb{N}_{\nu}^{T}\right\|_{2}\left\|\mathbb{P}_{\nu}\right\|_{2}$. But $\left\|\mathbb{N}_{\nu}^{T}\right\|_{2}=1$ (see the proof of Proposition 1) and from the definition of the matrix $\mathbb{P}_{\nu}$, it is clear that

$$
\left\|\mathbb{P}_{\nu}\right\|_{2} \leqslant\left(1+\|P\|_{2}^{2}\right)^{\frac{1}{2}} .
$$

From here, the conclusion follows.

This result shows that if $P$ is a point in $\mathbb{R}^{3}$ such that the numerical rank of $\mathbb{M}_{\nu}(P)$ drops, then $P$ has to be "close" to $\overline{\operatorname{Im}}(\phi)$ (notice that if $P$ is in a bounded box, then $\sigma_{1}$ is bounded above in terms of $\left.\|P\|_{2}\right)$. Moreover, it also shows that the more $P$ is singular (i.e. $r_{\epsilon}$ decreases), the more $P$ is close to $\overline{\operatorname{Im}}(\phi)$.

Notice, however, that Proposition 2 is not very helpful in practice because the constants $n_{1}$ and $c_{1}$ are difficult to predict (they follow from the Lojasiewicz inequalities). Nevertheless, it reinforces our experimental observations showing the very good numerical behavior of M-reps.

\subsection{Inversion through numerical computations}

Given a parameterization (1), we have shown in $\S 3.3$ that the inversion problem can be solved by computing a one-dimensional null space. Exploiting the capability of the SVD for computing numerical null space, as explained in $\$ 5.2$, it becomes possible to treat the inversion problem with numerical computations.

To be more precise, let $\mathbb{M}_{\nu}$ be an M-rep of a parameterization $\phi$, as in (1), and consider a given tolerance $\epsilon$. Let $P$ be a point in $\mathbb{R}^{3}$ that has a unique pre-image via $\phi$, i.e. such that $P=\phi\left(s_{0}\right)$ and $\operatorname{corank}\left(\mathbb{M}_{\nu}(P)\right)=1$. By Fact 3 , we know that $s_{0}$ can be extracted from the one-dimensional null space $\mathcal{N}_{m_{\nu}-1}\left(\mathbb{M}_{\nu}(P)^{T}\right)$ by a ratio computation (see $\S 3.3)$. We will denote by $u$ the vector spanning this null space, it is the last column of the matrix $U$ in the SVD of $\mathbb{M}_{\nu}(P)$.

Now, let $P_{\epsilon} \in \mathbb{R}^{3}$ be such that $\left\|P_{\epsilon}-P\right\|_{2} \leqslant \epsilon$, so that $r_{\epsilon}:=\operatorname{rank}\left(\mathbb{M}_{\nu}(P), \epsilon\right)<m_{\nu}$ by Corollary 1 . As explained in $\S 4.1$, determining the numerical rank $r_{\epsilon}$ is done by computing the SVD of $\mathbb{M}_{\nu}\left(P_{\epsilon}\right)=U_{\epsilon} \Sigma_{\epsilon} V_{\epsilon}^{T}$. Denote by $u_{\epsilon} \in \mathbb{R}^{m_{\nu}}$ the vector corresponding to the last column of the matrix $U_{\epsilon}$. By the discussion in $\S 5.2$, we deduce that

$$
\operatorname{dist}\left(u_{\epsilon}, u\right) \leqslant \frac{\sigma_{1}}{\sigma_{m_{\nu}-1}} \frac{\left\|\mathbb{M}_{\nu}\left(P_{\epsilon}\right)-\mathbb{M}_{\nu}(P)\right\|_{2}}{\sigma_{1}} \leqslant \frac{\sigma_{1}}{\sigma_{m_{\nu}-1}} \frac{\epsilon}{\sigma_{1}} .
$$

Therefore, it appears that if there is a distinct gap between $\sigma_{m_{\nu}}\left(\mathbb{M}_{\nu}\left(P_{\epsilon}\right)\right)$ and $\sigma_{m_{\nu}-1}\left(\mathbb{M}_{\nu}\left(P_{\epsilon}\right)\right)$, then the numerical rank 
is clearly equal to one and $u_{m_{\nu}}$ yields an accurate approximation of the null space of $\mathbb{M}_{\nu}(P)^{T}$. Moreover, if $\theta$ denotes the angle between $u_{\epsilon}$ and $u$, then $\operatorname{dist}\left(u_{\epsilon}, u\right)=\sin (\theta)$ by definition of this distance function (see $[17, \S 2.6 .3]$ ). It follows that the numerical values of the pre-image $s_{0}$ computed from the ratios of $u_{\epsilon}$ and $u$ are equal up to the numerical precision $\epsilon$.

Example 7 Take again the simple Example 2 of the unit sphere. If $P=(1 / \sqrt{3}, 1 / \sqrt{3}, 1 / \sqrt{3})$, then the exact null space of $\mathbb{M}_{1}(P)^{T}$ is generated by the vector

$$
\left[\begin{array}{c}
(\sqrt{3}-1) /(\sqrt{3}+1) \\
1 /(\sqrt{3}+1) \\
1 /(\sqrt{3}+1)
\end{array}\right] \approx\left[\begin{array}{l}
0.2679491925 \\
0.3660254037 \\
0.3660254037
\end{array}\right]
$$

where the numerical precision is 10 digits. Notice that we chose the generator of this null space which is normalized with respect to the 1-norm. This is because our M-rep has been built with Bernstein bases which form a partition of unity. Therefore, if $s_{0}=\left(u_{0}, v_{0}\right) \in \mathbb{R}^{2}$ is the pre-image of $P$ then (21) is equal to the vector

$$
\left[B_{0,0}^{1}\left(u_{0}, v_{0}\right), B_{0,1}^{1}\left(u_{0}, v_{0}\right)=v_{0}, B_{1,0}^{1}\left(u_{0}, v_{0}\right)=u_{0}\right]^{T} .
$$

It follows that

$$
u_{0}=v_{0}=1 /(\sqrt{3}+1) \approx 0.3660254037 .
$$

Now, we do the computations by using the numerical $M$ rep given in Figure 1 and take $P_{\epsilon}$ as the numerical approximation of $P$ with 10 digits precision. The computed singular values of $\mathbb{M}_{1}\left(P_{\epsilon}\right)$ are

$$
\sigma_{1}=0.7637626159, \sigma_{2}=0.4902332028, \sigma_{3}=2.3855877838 .10^{-10}
$$

and the last column of the matrix $U$ of the SVD is the 2norm unitary vector

\section{$\left[\begin{array}{lll}0.4597008431 & 0.6279630304 & 0.6279630302\end{array}\right]^{T}$}

By normalizing this vector with the 1-norm, we recover, as expected, the same approximation of $u_{0}$ and $v_{0}$ with 10 digits. Now, we add a perturbation of $10^{-5}$ to each coordinate of the point $P_{\epsilon}$. The singular values of $\mathbb{M}_{1}\left(P_{\epsilon}\right)$ become

$$
\sigma_{1}=0.7637701751, \sigma_{2}=0.4902374484, \sigma_{3}=0.0000114631
$$

and the last column of the matrix $U$ of the SVD is the 2norm unitary vector

\section{$\left[\begin{array}{lll}0.4596994681 & 0.6279635337 & 0.6279635335\end{array}\right]^{T}$}

By normalizing this vector with the 1-norm, we finally get

$$
u_{0} \approx 0.3660257759, v_{0} \approx 0.3660257758 .
$$

Comparing these values with (22), we check that up to 5 digits precision, the approximation of $u_{0}$ and $v_{0}$ are correct.

\section{The curve/M-rep intersection problem}

Our main motivation for introducing M-reps was to tackle intersection problems by generalizing the approach initiated by Canny and Manocha for non-singular matrix representations [23]. This approach consists in combining matrix-based implicit representations with generalized eigenvalues computations. We have extended it to M-reps recently: see $[22,10]$ for the curve/surface intersection and [10] for the curve/curve intersection by means of M-reps. Here, we quickly review how the properties of M-reps allow us to intersect a parameterized curve with another parameterized curve or surface that is represented by an M-rep, i.e. a curve/M-rep intersection problem.

For simplicity and clarity, let us consider the intersection between a ray and a parameterized surface (e.g. a Bezier patch obtained after the decomposition of a NURBS into Bézier patches [24, Chapter 5, §5.3]). This intersection problem is intensively used for ray tracing NURBS. A classical approach consists of computing an implicit representation of the ray as the intersection of two planes given by linear equations in $\mathbb{R}^{3}$. Then, substituting the surface parameterization into these two equations yields a polynomial system that can be solved via the Newton method. The intersection points are then obtained in the parameter space of the surface, so one can compute the normal of the surface at these points without difficulty (this is required for light reflection for instance).

Another way to proceed is to compute an implicit equation of the parameterized surface, to substitute the ray parameterization in this equation and solve a univariate polynomial. However, the implicitization step is very hard in general, due to the presence of base points. But M-reps have been designed to overcome this difficulty and are very easy to compute. So, let $\mathbb{M}_{\nu}$ be an M-rep of the surface parameterization. Substituting the ray parameterization into $\mathbb{M}_{\nu}$ yields a matrix whose entries are univariate linear polynomials in the parameter of the ray, say $t$. Finding the intersection points amounts to finding the values of $t$ such that this matrix is not full rank. These values are the generalized eigenvalues of this matrix (which is actually a univariate pencil of matrices) and, after a reduction step based on the SVD, they can be found by using algorithms that have been developed by the community of numerical linear algebra (see for instance [15, Chapter 4]). Using the ray parameterization, we then obtain the intersection points in $\mathbb{R}^{3}$. Now, thanks to the inversion property of M-reps, one can find the pre-image of these intersection points in the surface parameter space and hence compute the normal to the surface at these points (notice that intersection points that are singular points on the surface do not have a well defined normal vector). We refer the reader to [22] for the details (see also [1] for the linearization step in the Bernstein basis instead of in the power basis). Notice too that a study of the numerical accuracy in generalized eigenvalue computations can be found in [19]. In Figure 2, a ray tracing of the sphere given in Example 2 is shown. It has been obtained by Valentin Michelet (an engineering student) who developed his own ray tracer from scratch by computing the ray/surface intersection by means of M-reps. Two other illustrations are given in Figure 3.

It should be clear to the reader that the above method 


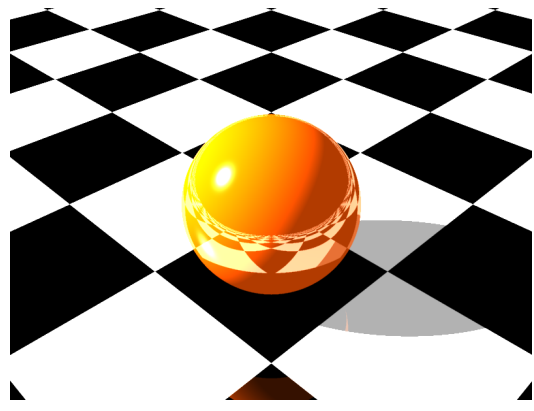

Fig. 2. Ray tracing of the sphere by means of M-reps

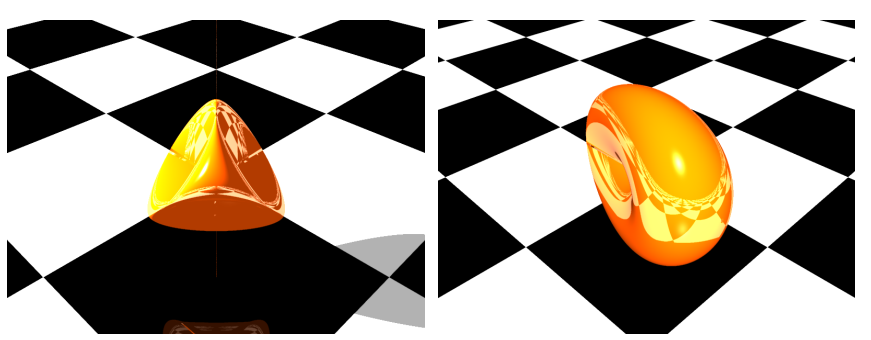

Fig. 3. Ray tracing of Steiner surfaces by means of M-reps

can be applied with any parameterization that admits an M-rep. Therefore, parameterized curve/curve and parameterized curve/surface intersections can be treated exactly in the same way. An implementation of these intersections has been done in the algebraic geometric modeler Axel, in the plugin Shape; it is freely available at the URL: http: //axel.inria.fr.

\section{Conclusion}

In this paper, a new implicit representation of a parameterized curve or surface is presented: a matrix whose entries depend on the space variables. It has the property that its rank drops after evaluation at a given point if and only if this point belongs to the curve or surface under consideration. The main properties of such an implicit matrix representation are described and a detailed analysis of its numerical behavior is given. Its use for solving intersection problems is also illustrated and seems to be promising. The surface/surface intersection problem by means of this new representation is still a topic for future research. A preliminary investigation has already started [11], but more work is necessary to get a numerically stable and robust method, as we provided for the curve/M-rep intersection problem.

\section{References}

[1] A. Amiraslani, R. M. Corless, and P. Lancaster. Linearization of matrix polynomials expressed in polynomial bases. IMA J. Numer. Anal., 29(1):141-157, 2009.

[2] Oliver J. D. Barrowclough and Tor Dokken. Approximate implicitization using linear algebra. J. Appl. Math., pages Art. ID 293746, 25, 2012.

[3] Edward Bierstone and Pierre D. Milman. Semianalytic and subanalytic sets. Inst. Hautes Etudes Sci. Publ. Math., (67):542, 1988.
[4] Nicolás Botbol. The implicit equation of a multigraded hypersurface. J. Algebra, 348:381-401, 2011.

[5] Nicolás Botbol, Laurent Busé, and Marc Chardin. Fitting ideals and multiple-points of surface parameterizations. In preparation, 2013.

[6] Laurent Busé and Marc Chardin. Implicitizing rational hypersurfaces using approximation complexes. J. Symbolic Comput., 40(4-5):1150-1168, 2005.

[7] Laurent Busé, Marc Chardin, and Jean-Pierre Jouanolou. Torsion of the symmetric algebra and implicitization. Proceedings of the American Mathematical Society, 137(06):1855-1865., February 2009.

[8] Laurent Busé, David Cox, and Carlos D'Andrea. Implicitization of surfaces in $\mathbb{P}^{3}$ in the presence of base points. J. Algebra Appl., 2(2):189-214, 2003.

[9] Laurent Busé and Jean-Pierre Jouanolou. On the closed image of a rational map and the implicitization problem. J. Algebra, 265(1):312-357, 2003.

[10] Laurent Busé and Thang Luu Ba. Matrix-based Implicit Representations of Rational Algebraic Curves and Applications. Computer Aided Geometric Design, 27(9):681-699, 2010.

[11] Laurent Busé and Thang Luu Ba. The surface/surface intersection problem by means of matrix based representations. Comput. Aided Geom. Design, 29(8):579-598, 2012.

[12] Eng-Wee Chionh and Ronald N. Goldman. Degree, multiplicity, and inversion formulas for rational surfaces using u-resultants. Computer Aided Geometric Design, 9(2):93 - 108, 1992.

[13] David Cox, Ronald Goldman, and Ming Zhang. On the validity of implicitization by moving quadrics of rational surfaces with no base points. J. Symbolic Comput., 29(3):419-440, 2000.

[14] David Cox, John Little, and Donal O'Shea. Using algebraic geometry, volume 185 of Graduate Texts in Mathematics. Springer-Verlag, New York, 1998.

[15] James W. Demmel. Applied numerical linear algebra. Society for Industrial and Applied Mathematics (SIAM), Philadelphia, PA, 1997.

[16] Tor Dokken. Approximate implicitization. In Mathematical methods for curves and surfaces (Oslo, 2000), Innov. Appl. Math., pages 81-102. Vanderbilt Univ. Press, 2001.

[17] Gene H. Golub and Charles F. Van Loan. Matrix computations. Johns Hopkins Studies in the Mathematical Sciences. Johns Hopkins University Press, Baltimore, MD, third edition, 1996.

[18] Per Christian Hansen. Rank-deficient and discrete ill-posed problems. SIAM Monographs on Mathematical Modeling and Computation. Society for Industrial and Applied Mathematics (SIAM), 1998.

[19] Y. Hua and T. Sarkar. On SVD for estimating generalized eigenvalues of singular matrix pencil in noise. IEEE Transactions on Signal Processing, 39(4):892-900, 1991.

[20] Amit Khetan and Carlos D'Andrea. Implicitization of rational surfaces using toric varieties. J. Algebra, 303(2):543-565, 2006.

[21] János Kollár. An effective łojasiewicz inequality for real polynomials. Period. Math. Hungar., 38(3):213-221, 1999.

[22] Thang Luu Ba, Laurent Busé, and Bernard Mourrain. Curve/surface intersection problem by means of matrix representations. In $\mathrm{H}$. Kai and H. Sekigawa, editors, $S N C$ Conference, pages 71-78. ACM Press, 2009.

[23] Dinesh Manocha and John Canny. A new approach for surface intersection. In Proceedings of the first ACM symposium on Solid modeling foundations and CAD/CAM applications, pages 209-219, Austin, Texas, United States, 1991. ACM.

[24] Les A. Piegl and Wayne Tiller. The NURBS book (2. ed.). Monographs in visual communication. Springer, 1997.

[25] T.W. Sederberg and F. Chen. Implicitization using moving curves and surfaces. In Proceedings of SIGGRAPH, volume 29, pages 301-308, 1995.

[26] Pablo Solernó. Effective łojasiewicz inequalities in semialgebraic geometry. Appl. Algebra Engrg. Comm. Comput., 2(1):2-14, 1991. 